\section{JTI}

JOURNAL OF

TRAUMA AND INJURY

\title{
Laparoscopy in Blunt Abdominal Trauma: Diaphragmatic and Bladder Lacerations Repair
}

\author{
Ruben Martins, M.D., Martins dos Santos, M.D., Tatiana Revez, M.D. \\ General Surgery Department, Faro Hospital, Algarve Hospital University Center, Faro, \\ Portugal
}

Received: June 24, 2019

Revised: August 2, 2019

Accepted: August 2, 2019

Correspondence to

Ruben Martins, M.D.

General Surgery Department, Faro Hospital, Algarve Hospital University Center, Rua Leão Penedo, Faro 8000-386, Portugal Tel: +351-936-790-391

E-mail: rubenafpmartins@gmail.com

The growing use of laparoscopy in elective surgery has led to its increase utilization in emergency surgery. However, the employment of laparoscopy in abdominal trauma is still unusual. Here in we report a case of a patient with blunt abdominal trauma that resulted in a combination of exceptional traumatic lesions, diaphragmatic and bladder lacerations. Both injuries were diagnosed and successfully resolved by laparoscopy. The report of this type of lesions and resolution is extremely rare, being this the second case described in the international literature. This article intends to show that laparoscopy may not only be used as a diagnostic tool, but also as a therapeutic instrument in selected cases of blunt abdominal trauma.

Keywords: Trauma; Laparoscopy; Diaphragm; Bladder

\section{INTRODUCTION}

Laparoscopy has been gradually accepted as a diagnostic tool in abdominal trauma, being generally considered for penetrating abdominal trauma [1]. Its use as a therapeutic instrument, particularly in blunt abdominal trauma, is less common, existing few publications reporting this matter, being the majority case reports. The clinical conditions that are generally accepted for laparoscopy are stable patients with unclear intra-abdominal fluid and abdominal pain. We present such a case that resulted in a rare combination of traumatic injuries.

This is an Open Access article distributed under the terms of the Creative Commons Attribution Non-Commercial License (http://creativecommons.org/licenses/by-nc/4.0/) which permits unrestricted noncommercial use, distribution, and reproduction in any medium, provided the original work is properly cited. 


\section{CASE REPORT}

A 49 year-old female patient admitted to the emergency room after a car accident, with frontal crash and high kinetic energy. The patient complains were loss of consciousness and pain in the head, right chest, abdomen and limbs, right upper and left lower limb. She was hemodynamically stable, without dyspnea and had an oxygen saturation of $96 \%$ without oxygen supplementation. She was disoriented, with a Glasgow coma scale of 14. There were no changes in cardiac and pulmonary auscultation. On inspection a deformation and bruise were evident at the right lower hemithorax, with pain at palpation. The abdomen was without visible lesions. With abdominal palpation the patient presented diffuse tenderness at the superficial and deep palpation, mainly at the lower abdomen. A deformation on the left tibiotarsal joint was patent. A urethral catheter was placed, having drained bloodstained urine.

Head computed tomography (CT) scan showed no lesions, limbs $\mathrm{X}$-ray revealed a left ankle and right wrist fractures. The thoraco-abdominal CT scan demonstrated bilateral costal arch fractures, pulmonary contusion, minimum bilateral pleural effusion, abdominal wall hernia with small bowel in the left flank, peri-hepatic, peri-splenic and pelvic peritoneal effusion (Fig. 1).

The patient mentioned that the abdominal hernia appeared just after the accident, and it was painless. After excluding an intracranial lesion and in the presence of abdominal pain with intra-abdominal fluid in a stable
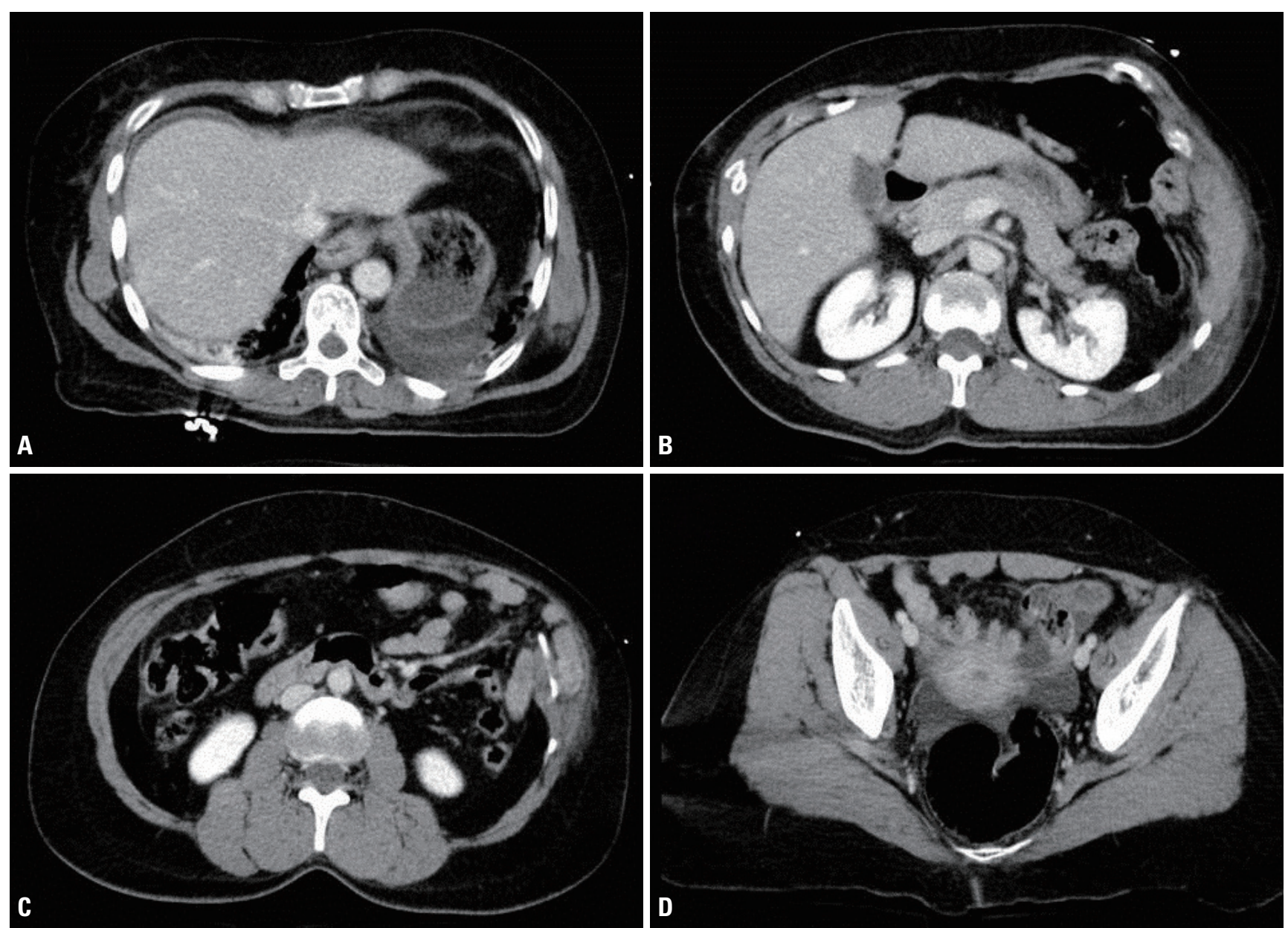

Fig. 1. Thoraco-abdominal computed tomography scan. (A) Hydrothorax with peri-hepatic and peri-splenic peritoneal effusion. (B, C) Left abdominal wall hernia with small bowel. (D) Pelvic peritoneal effusion. 


\section{JTI}

patient victim of blunt abdominal trauma we proposed a diagnostic laparoscopy that was accepted by the patient.

In the operating room it was given cefoxitin $2 \mathrm{~g}$ at anesthetic induction. The patient was placed in a supine position, legs together and arms at a $90^{\circ}$ angle to the body. Surgeon and assistant were positioned on the right side of the patient. A $10-\mathrm{mm}$ trocar was inserted via a paraumbilical incision. The pneumoperitoneum was created at $12 \mathrm{mmHg}$ pressure. A $30^{\circ}$ degree angle $10 \mathrm{~mm}$ laparoscope was used for abdominal exploration. It identified a clear intra-abdominal fluid in moderate volume, and a diaphragmatic and bladder lacerations. The diaphragmatic laceration was a grade IV lesion [2] with $15-20 \mathrm{~cm}$ of greater axis, from the splenic region to the implantation area of the diaphragm. Small bowel was at the laceration and beyond the costal grid, being evident a costal fracture in the middle of the intestinal loops (Fig. 2). The left thorax cavity was inspected without obvious lesions. The bladder wall laceration was a grade IV lesion [2], at its dome and with $10 \mathrm{~cm}$ of greater axis (Fig. 3). Additional ports were placed, a $10 \mathrm{~mm}$ port at right iliac fossa and a $5 \mathrm{~mm}$ port at the right upper quadrant. No other lesions were identified, including in the small bowel that was near the costal fracture. The bladder lesion was resolved with a continuous suture of 00 absorbable thread. We then performed a test to the closure by filling the bladder with a diluted povidone iodine solution, without leakage.

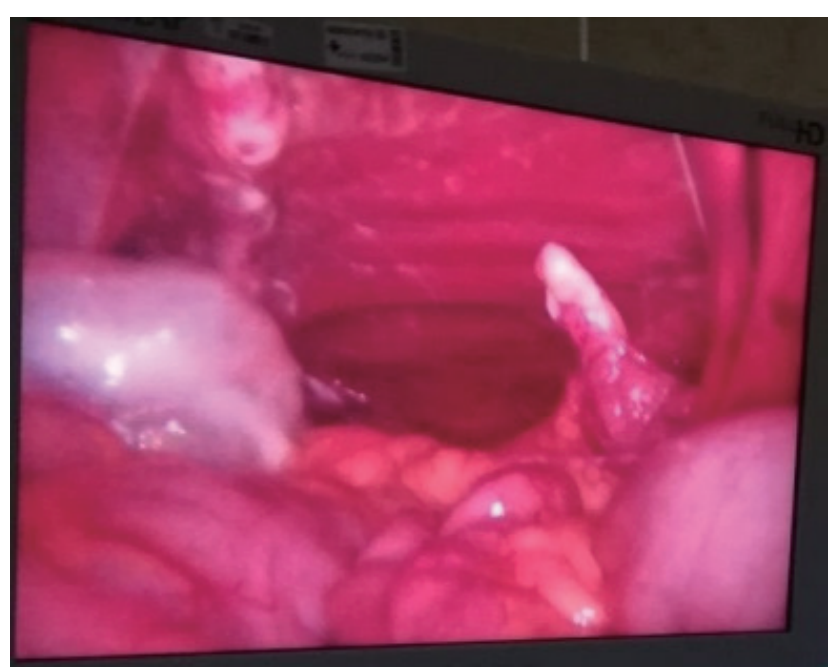

Fig. 2. Per-operative photography showing the diaphragm laceration with the thoracic wall behind and a costal fracture.
An extra 5-mm port was placed in the left upper quadrant and a $10 \mathrm{~mm}$ port was placed at the epigastric region, in order to realize the repair of the diaphragmatic rupture. A continuous suture with interval interrupted suture was done with 00 absorbable thread, in order to share the pressure between points (Fig. 4). The surgery was performed without intercurrences and without evident blood loss. Abdominal and bilateral thoracic drains were placed. The patient was hospitalized in the intensive care unit for 30 days. The transfer for the ward was delayed due to difficulties in extubation. The abdominal drain was removed in the third postoperative day and the thoracic

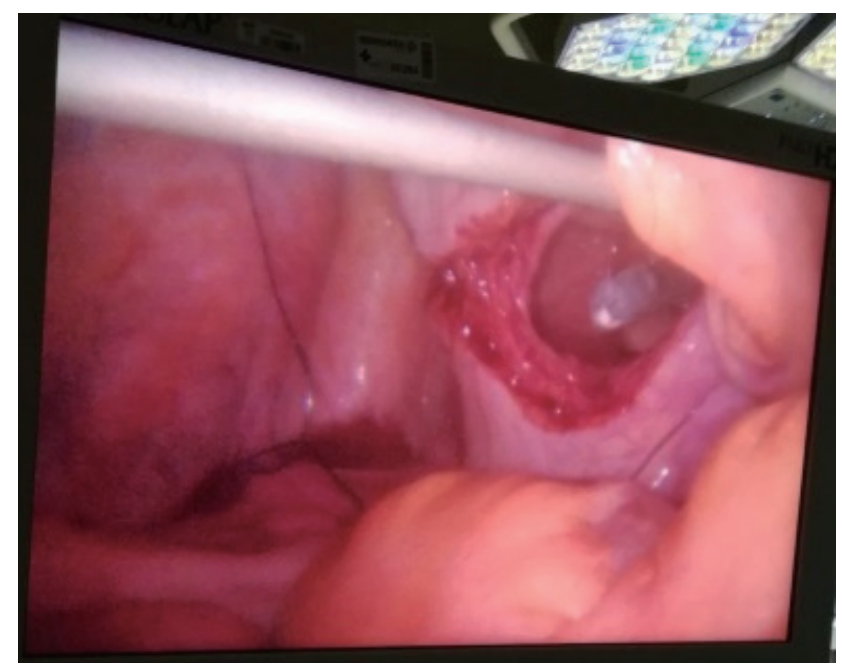

Fig. 3. Per-operative photography showing the defects in the urinary bladder.

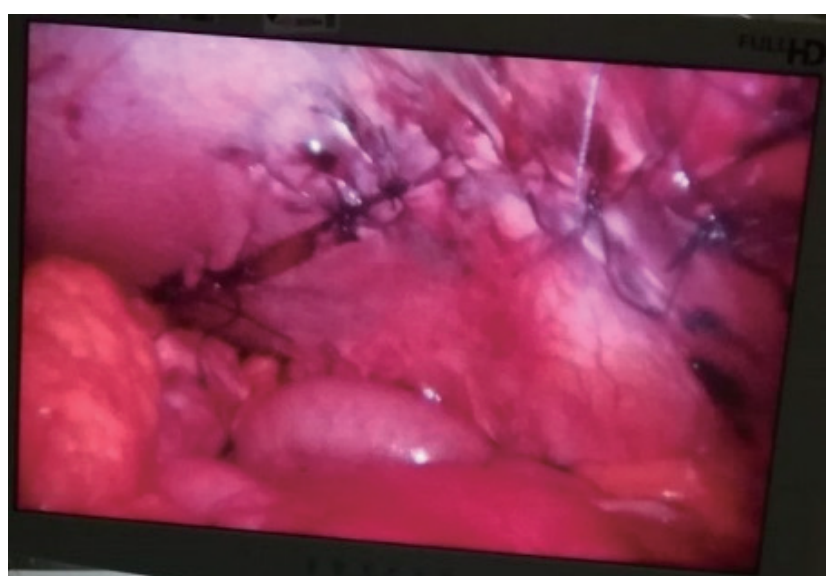

Fig. 4. Per-operative photography showing laparoscopic repair of the diaphragm. 
drains were removed nine days after surgery. The urethral catheter was removed three weeks after the operation. Succeeding the patient stabilization, she was transferred to the orthopedic department for continuous treatment.

Three months after the accident the patient was re-assessed by the trauma surgeon that performed the surgery. She was well, without abdominal or chest pain and without respiratory complains. One year after the accident and consequent surgery, the patient remained well, without complaints.

\section{DISCUSSION}

Laparoscopy is an attractive tool in selected abdominal trauma [3]. It has high sensitivity and specificity (99\% and $100 \%$ respectively) in the diagnosis of abdominal lesions related to trauma, subsequently it has an important role in avoiding non-therapeutic laparotomies [1,3]. With the accumulated experience in advanced laparoscopic procedures, the laparoscopy in trauma evolved not just for diagnosis proposes, but also to perform therapeutic procedures. A recent study defends the therapeutic laparoscopy in blunt abdominal trauma with high success rate (about 92\%), when the patient is hemodynamically stable and in some select scenarios which are suspected hollow visceral injuries, suspected diaphragm injuries, failed non-operative management of liver or spleen injuries and isolated intra-abdominal fluid with clinical findings [1]. Other studies have a lower level of success, probably related to the incorrectly selected cases or the low experience or laparoscopy skills of the surgeons involved.

The incidence of diaphragmatic rupture in blunt abdominal trauma varies between $1 \%$ and $7 \%$ in hospitalized victims of motor vehicle crashes [4]. The diagnostic success rate fluctuates between $25 \%$ to $50 \%$ in chest X-ray and $42 \%$ to $84 \%$ in CT scan [4]. Several authors report high diagnostic accuracy with laparoscopy, and the present case is representative of that accuracy. Laparoscopy also permits the exploration of the thoracic cavity, which is extremely difficult with laparotomy, and may show unpredictable lesions.

Bladder rupture occurs in about $2 \%$ of all abdominal trauma requiring surgery, and in $40 \%$ of all cases the laceration opens to the abdominal cavity [4]. Usually the rupture takes place at its dome, which is the most vulnerable part of the organ when it is full. The principal signs of bladder rupture are suprapubic discomfort and hematuria. CT scan has an estimated accuracy of $61 \%$. Cystography has better results, about $95 \%$ of sensitivity and $100 \%$ of specificity, but demands time and available technicians. In the current case a traumatic bladder injury was not evident on CT scan, but the clinic presentation and the intra-abdominal fluid raised suspicion, which was confirmed by laparoscopy.

This case shows that laparoscopy can identify lesions that the CT scan cannot. Even a specific organ exam like cystography, that could identify the bladder lesion, could be helded if laparoscopy did not find the lesion. In this case the cystography was going to be time consuming and was not going to change the need for surgery. It would be used if no lesion was found and the suspicion for a urinary lesion remained.

The hernia revealed by the objective examination and the CT scan was in fact a combination of the diaphragm laceration with the costal arch fractures, that breached the thoracoabdominal wall. This was repaired by the closure of the diaphragm laceration. The diaphragm laceration dimension recommended a mesh application, but there was none available in the hospital. Therefore, a continuous and interrupted suture was done, associated with a controlled mechanical ventilation after surgery, in order not to overcharge the diaphragm suture. Although non-absorbable threads are preferable in these situations, slowly absorbable threads, as the one used, has shown similar results $[5,6]$.

In addition to diagnosing traumatic lesions, laparoscopy allows performing therapeutic procedures. This case is an example of this possibility, with the realization of laparoscopic suture of both lacerations. This technique allows the repairing of structures that limit the abdominal cavity superiorly and inferiorly, which avoid a large abdominal incision, in this case a xiphisternum to symphysis pubis incision, with its possible related complications.

It is also important to have a dedicated and thorough medical and nursing staff in order to have all the endurance and material that an emergent laparoscopic procedure may require. Up to our knowledge this is only the 
second reported case of a successful laparoscopic repair of a diaphragmatic and bladder injuries and confirms the efficiency of laparoscopy in the diagnosis and treatment of patients with blunt abdominal trauma [7].

\section{CONCLUSION}

As well as laparoscopy had revolutionized elective surgery, it is time for this technique to improve the emergent trauma surgery, in order to upgrade the emergency health care. It has taken slow but consistent steps in this matter, and has showed its ability to identify and resolve innumerable abdominal conditions, providing good recovery for the patients. The trauma patients that clearly benefit from laparoscopy are the ones with suspected or confirmed intra-abdominal lesion that maintain hemodynamic stability. The intent of laparoscopy should be to diagnose a lesion and treat it when indicated. It is also important to continue developing the surgeon's laparoscopic skills in order to offer the best possible care.

\section{REFERENCES}

1. Lin HF, Chen YD, Chen SC. Value of diagnostic and therapeutic laparoscopy for patients with blunt abdominal trauma: a 10year medical center experience. PLoS One 2018;13:e0193379.

2. Moore EE, Cogbill T, Malangoni M, Jorkovich G, Champion H. Scaling system for organ specific injuries. Curr Opin Crit Care 1996;2:450-62.

3. Lim KH, Chung BS, Kim JY, Kim SS. Laparoscopic surgery in abdominal trauma: a single center review of a 7-year experience. World J Emerg Surg 2015;10:16.

4. Gourgiotis S, Rothkegel S, Germanos S. Combined diaphragmatic and urinary bladder rupture after minor motorcycle accident (report of a case and literature review). Ulus Travma Acil Cerrahi Derg 2008;14:163-6.

5. Matsevych OY. Blunt diaphragmatic rupture: four year's experience. Hernia 2008;12:73-8.

6. Ties JS, Peschman JR, Moreno A, Mathiason MA, Kallies KJ, Martin RF, et al. Evolution in the management of traumatic diaphragmatic injuries: a multicenter review. J Trauma Acute Care Surg 2014;76:1024-8.

7. Gandhi RK, Dhandapani BS, Barvadheesh RC. Combination injuries of diaphragm and urinary bladder resulting urinothorax. J Minim Access Surg 2015;11:149-50. 